\title{
ON THE ZETA FUNCTIONS OF PREHOMOGENEOUS VECTOR SPACES FOR PAIR OF SIMPLE ALGEBRAS
}

\author{
TAKASHI TANIGUCHI
}

\begin{abstract}
In this paper, we consider the prehomogeneous vector space for pair of simple algebras which are $k$-forms of the $D_{4}$ type and the $E_{6}$ type. We mainly study the non-split cases. The main purpose of this paper is to determine the principal parts of the global zeta functions associated with these spaces in the non-split cases. We also give a description of the sets of rational orbits of these spaces, which suggests the expected density theorems arising from the properties of these zeta functions.
\end{abstract}

\section{INTRODUCTION}

Let $k$ be a field and $D$ a simple algebra of dimension 4 or 9 over $k$. We denote by $D^{\text {op }}$ the opposite algebra of $D$. In this paper, we consider the prehomogeneous vector space $(G, V)=(G, \rho, V)$ where

$$
G=D^{\times} \times\left(D^{\mathrm{op}}\right)^{\times} \times \mathrm{GL}(2), \quad V=D \otimes k^{2},
$$

and

$$
\rho(g)(a \otimes v)=\left(g_{11} a g_{12}\right) \otimes\left(g_{2} v\right) \quad \text { for } \quad g=\left(g_{11}, g_{12}, g_{2}\right) \in G, a \in D, v \in k^{2} .
$$

We call the prehomogeneous vector space $D_{4}$ type and $E_{6}$ type if the dimension of $D$ is 4 and 9 , respectively. This representation is a $k$-form of

$$
G^{\prime}=\mathrm{GL}(n) \times \mathrm{GL}(n) \times \mathrm{GL}(2), \quad V^{\prime}=k^{n} \otimes k^{n} \otimes k^{2}
$$

for $n=2$ and $n=3$ if the dimension of $D$ is 4 and 9 , respectively. If $D$ is split, then $(G, V)$ is equivariant to $\left(G^{\prime}, V^{\prime}\right)$ over $k$. In this paper, we give a certain description of $k$-rational orbits and determine the structure of the stabilizers for semi-stable points. Also we determine the principal parts of the global zeta function for the non-split cases of $(G, V)$ over an algebraic number field $k$.

We recall the definition of prehomogeneous vector spaces. Here we give a definition of a certain restricted class instead of giving general one for simplicity.

Definition 1.1. An irreducible representation of a connected reductive group $(G, V)$ over $k$ is called a prehomogeneous vector space if

(1) there exists a Zariski open $G$-orbit in $V$ and

(2) there exists a non-constant polynomial $P \in k[V]$ and a rational character $\chi$ of $G$ such that $P(g x)=\chi(g) P(x)$ for all $g \in G$ and $x \in V$.

Irreducible prehomogeneous vector spaces over an arbitrary characteristic 0 algebraically closed field was classified by Sato and Kimura in [14. Sato and Shintani

Date: November 16, 2018.

Key words and phrases. prehomogeneous vector space, global zeta function, simple algebra, density theorem. 
[15] defined global zeta functions for prehomogeneous vector spaces if $(G, V)$ is defined over a number field.

The information of the principal part at the rightmost pole of the global zeta function for a prehomogeneous vector space together with an appropriate local theory yields interesting density theorems. For example, using Shintani's result [16] for the space of binary cubic forms, Datskovsky and Wright 3, 4, gave the zeta function theoretic proof of the Davenport and Heilbronn [5, 6] density theorem

$$
\sum_{\substack{[F: \mathbb{Q}]=3 \\\left|\Delta_{F}\right| \leq x}} 1 \sim \frac{x}{\zeta(3)} \quad(x \rightarrow \infty),
$$

where $F$ runs through all the cubic fields with the absolute value of its discriminant $\left|\Delta_{F}\right|$ is not bigger than $x$. Also recent works concerning to the space of pairs of binary Hermitian forms [8, 9, 10] by Kable and Yukie gave certain new density theorems together with Yukie's global theory [21]. For the statement of the density theorem, see the introduction of $\left[8\right.$. Note that this case is another $k$-form of the $D_{4}$ case. These $k$-forms are listed in H. Saito's classification [12].

We return to our prehomogeneous vector space (1.1). The following theorem is a main result of this paper.

Theorem 1.2. Let $D$ be a non-split simple algebra of dimension $m=4$ or 9 . The global zeta function $Z(\Phi, s)$ associated with the prehomogeneous vector space (1.1) can be continued meromorphically to the region $\Re(s)>2 m-2$ only with a possible simple pole at $s=2 m$ with the residue $\tau\left(G_{1}\right) \mathfrak{V}_{2} \int_{V_{\mathbb{A}}} \Phi(x) d x$.

The constants $\tau\left(G_{1}\right), \mathfrak{V}_{2}$ and the measure $d x$ on $V_{\mathbb{A}}$ are defined in Section 4 All the other poles are also described by means of certain distributions in Theorem 4.24, but the above theorem is enough to get the density theorems. On the other hand, the expected density theorems require not only the standard tauberian theorem, but also the appropriate local theory and what is called "filtering process", which will be studied in a forthcoming paper. For a general transition process from the tauberian theorem for global zeta function of prehomogeneous vector spaces to density theorems, see [20].

The expected density theorems from our cases will be discussed in Remark 3.10 using a result in Section 3 , but we also give a brief summary here. We assume $k=\mathbb{Q}$ for simplicity. For a finite extension $F$ of $\mathbb{Q}, h_{F}$ and $R_{F}$ denote the class number and the regulator of $F$, respectively.

For a prehomogeneous vector space $(G, V)$ in Definition 1.1. set $V_{\mathbb{Q}}^{\text {ss }}=\left\{x \in V_{\mathbb{Q}}\right.$ $P(x) \neq 0\}$ where $P(x)$ is as in $(2)$ and $\widetilde{T}=\operatorname{ker}(G \rightarrow \mathrm{GL}(V))$. For $x \in V_{\mathbb{Q}}^{\text {ss }}, G_{x}$ denotes the stabilizer of $x$ and $G_{x}^{\circ}$ denotes its identity component. Roughly speaking, the global zeta function is a counting function for the unnormalized Tamagawa numbers of $G_{x}^{\circ} / \widetilde{T}$ of points in $x \in G_{\mathbb{Q}} \backslash V_{\mathbb{Q}}^{\text {ss }}$ (see [20], for example). Hence, by Proposition 3.6] and 3.7, the behavior of the zeta function at the rightmost pole for the $D_{4}$ case and the $E_{6}$ case (both split and non-split) yields the asymptotic behavior of the following function

$$
\sum_{\substack{[F: \mathbb{Q}]=2 \\\left|\Delta_{F}\right| \leq x \\ \text { mbeddable into } D}} h_{F}^{2} R_{F}^{2} \quad \text { and } \quad \sum_{\substack{[F: \mathbb{Q}]=3 \\\left|\Delta_{F}\right| \leq x \\ F \text { is embeddable into } D}} h_{F} R_{F}
$$

as $x \rightarrow \infty$, respectively. If the method of filtering process in [3, 4, [2], [8, 9, 10] is applicable to compute the above density, one can also compute a similar density with 
finitely many local conditions by the same method. It is also easy to show that for each $D$, the condition $F \subset D$ can be determined by finitely many local conditions of $F$. Hence, the expected density theorems from the non-split cases seems to be quite similar to that of from the split cases.

One advantage of non-split cases is that the global theory becomes much easier. The analysis of the global zeta function becomes much complicated as the split rank of the group growth, and we could not yet success to establish the global theory for the split $D_{4}$ and $E_{6}$ cases. Especially the rank of the group is 5 for the split $E_{6}$ case, and the complexity of computing the principal part of the zeta function seems to be formidable.

For the rest of this section, we will give the contents of this paper and the notations used in this paper. More specific notations will be introduced in each section. In Section 2. we will define the space of pair of simple algebras and summarize its basic properties. Before starting the global theory in Section 4. we will give a certain description of rational orbits in Section 3. The split cases are treated in [19, §3], and this is a slight generalization of those cases. We prove that the set of rational orbits corresponds one to one to a certain set of quadratic extensions and cubic extensions for the $D_{4}$ case and the $E_{6}$ case, respectively. Also we determine the structure of the stabilizers for semi-stable points. The expected density theorems from our cases will be discussed in Remark 3.10.

In Section 4, we study the global theory for the non-split cases. In \$4.1, we introduce notations used in this section and review some basic facts on adelic analysis. In 4.2 we define a global zeta function. Also we will give an estimate of an incomplete theta series. Although H. Saito [13] proved the convergence of the all global zeta functions associated with prehomogeneous vector spaces, we need the estimate in order to use Shintani's lemma. In 4.3 , we divide the global zeta function into the "entire part" and the "principal part" by using the Poisson summation formula. We study the "principal part" in later subsections.

In 4.4, we introduce a stratification of unstable points. To separate the contribution from unstable strata, we use Shintani's lemma. In \$4.5, we review Shintani's lemma and apply it to our cases. Since $D^{\times}$is of rank 0, the smoothed Eisenstein series for our case is essentially the same as that of GL(2). In \$4.6, we review some analytic properties of the zeta function associated with (single) simple algebra, because this zeta function appears in the induction process. In $\$ 4.7$, we compute contributions from unstable points. By putting the results together we have obtained in 4.4-44.7, we determine the principal part of the global zeta function in $\$ 4.8$

The standard symbols $\mathbb{Q}, \mathbb{R}, \mathbb{C}$ and $\mathbb{Z}$ will denote respectively the rational, real and complex numbers and the rational integers. If $R$ is any ring then $R^{\times}$is the set of invertible elements of $R$ and if $V$ is a variety defined over $R$ then $V_{R}$ denotes its $R$-points. In Section 2 and 3, $k$ denotes arbitrary field. In Section 4, $k$ denotes an algebraic number field.

Acknowledgments. This work is the author's doctor's thesis at University of Tokyo. The author would like to express his sincere gratitude to his advisor T. Terasoma for the constant support, encouragement and many helpful suggestions. The author would like to heartily thank Professor A. Yukie for the encouragement and many invaluable inspiring suggestions. The author is deeply grateful to Professor T. Oda for the support and consultations. He also would like to thank his colleague T. Ito with the many useful discussions. This work is partially supported by 21st Century (University of Tokyo) COE program, of Ministry of Education, Culture, Sports, Science and Technology. 


\section{THE SPACE OF PAIR OF SIMPLE ALGEBRAS}

In this section, we define the representation of the space of pair of simple algebras, and discuss its basic properties.

Let $k$ be an arbitrary field and $D$ a simple algebra over $k$ of dimension $m=n^{2}, n \geq 1$. Let $\mathcal{T}$ and $\mathcal{N}$ be the reduced trace and reduced norm, respectively. We denote by $D^{\text {op }}$ the opposite algebra of $D$. We introduce a group $G_{1}$ and its representation space $W$ as follows. Let

$$
G_{1}=D^{\times} \times\left(D^{\mathrm{op}}\right)^{\times}
$$

That is, $G_{1}$ is equal to $D^{\times} \times D^{\times}$set theoretically and the multiplication law is given by $\left(g_{11}, g_{12}\right)\left(h_{11}, h_{12}\right)=\left(g_{11} h_{11}, h_{12} g_{12}\right)$. We regard $G_{1}$ as an algebraic group over $k$. The simple algebra $D$ can be considered as a vector space over $k$. When we regard $D$ as a vector space over $k$, we denote this space as $W$. We define the action of $G_{1}$ on $W$ as follows:

$$
\left(g_{1}, w\right) \longmapsto g_{11} w g_{12}, \quad g_{1}=\left(g_{11}, g_{12}\right) \in G_{1}, w \in W .
$$

This defines a representation $W$ of $G_{1}$. Clearly, $\left(G_{1}, W\right)$ is a prehomogeneous vector space. We discuss the properties of the zeta function associated with this space in 4.6 , which will be used in the analysis of the zeta function associated with the space of pair of simple algebras.

Let $G_{2}=\mathrm{GL}(2)$ and $k^{2}$ the standard representation of $G_{2}$. The group $G=G_{1} \times G_{2}$ acts naturally on $V=W \otimes k^{2}$. This is a $k$-form of $\left(\mathrm{GL}(n) \times \mathrm{GL}(n) \times \mathrm{GL}(2), k^{n} \otimes k^{n} \otimes k^{2}\right)$, and it is proved in [14] that this is a prehomogeneous vector space if and only if $n=2$ or $n=3$. Since we are interested in prehomogeneous vector space, we consider the case $n=2,3$ for the rest of this paper. That is, $D$ is a simple algebra of dimension 4 or 9 . We call these representation $D_{4}$ type and $E_{6}$ type for $n=2$ and $n=3$, respectively following [19].

We describe the action more explicitly. Throughout of this paper, we express elements of $V \cong W \oplus W$ as $x=\left(x_{1}, x_{2}\right)$. We identify $x=\left(x_{1}, x_{2}\right) \in V$ with $x(v)=v_{1} x_{1}+v_{2} x_{2}$ which is an element of simple algebra with entries in linear forms in two variables $v=$ $\left(v_{1}, v_{2}\right)$. Then the action of $g=\left(g_{11}, g_{12}, g_{2}\right) \in G$ on $x \in V$ is defined by

$$
(g x)(v)=g_{11} x\left(v g_{2}\right) g_{12} \text {. }
$$

We put $F_{x}(v)=\mathcal{N}(x(v))$. This is a binary quadratic form (resp. cubic form) in variables $v=\left(v_{1}, v_{2}\right)$ if $n=2$ (resp. $\left.n=3\right)$, and the discriminant $P(x)(x \in V)$ is a polynomial in $V$. The polynomial $P(x)$ is characterized by

$$
P(x)=\prod_{i<j}\left(\alpha_{i} \beta_{j}-\alpha_{j} \beta_{i}\right)^{2} \quad \text { for } \quad F_{x}(v)=\prod_{1 \leq i \leq n}\left(\alpha_{i} v_{1}-\beta_{i} v_{2}\right), \quad x \in V_{\bar{k}} .
$$

Let $\chi_{i}(i=1,2)$ be the character of $G_{i}$ defined by

$$
\chi_{1}\left(g_{1}\right)=\mathcal{N}\left(g_{11}\right) \mathcal{N}\left(g_{12}\right), \quad \chi_{2}\left(g_{2}\right)=\operatorname{det} g_{2},
$$

respectively. We define $\chi(g)=\chi_{1}\left(g_{1}\right)^{2} \chi_{2}\left(g_{2}\right)^{2}$ for $n=2$ and $\chi(g)=\chi_{1}\left(g_{1}\right)^{4} \chi_{2}\left(g_{2}\right)^{6}$ for $n=3$. Then one can easily see that

$$
P(g x)=\chi(g) P(x)
$$

and hence $P(x)$ is a relative invariant polynomial with respect to the character $\chi$. Let $S=\{x \in V \mid P(x)=0\}$ and $V^{\text {ss }}=\{x \in V \mid P(x) \neq 0\}$ and call them the set of unstable points and semi-stable points, respectively. That is, $x \in V$ is semi-stable if and only if $F_{x}(v)$ does not have a multiple root in $\mathbb{P}^{1}=\left\{\left(v_{1}: v_{2}\right)\right\}$. 


\section{RATIONAL ORBIT DECOMPOSITION}

$\S 3.1$. Rational orbit decomposition. In this section, we will interpret the rational orbit space $G_{k} \backslash V_{k}^{\text {ss }}$ and determine the structure of the stabilizers for semi-stable points. The split cases are treated in [19, §3], and here is a slight generalization of that. For the expected density theorems, see Remark 3.10. For $x \in V_{k}^{\text {ss }}$, let $G_{x}$ be the stabilizer of $x$ and $G_{x}^{\circ}$ its identity component.

Let $\mathfrak{Q}_{k}^{\text {sep }}$ (resp. $\mathfrak{C}_{k}^{\text {sep }}$ ) be set of isomorphism classes of separable commutative $k$-algebras of dimension 2 (resp. 3). For example, $\mathfrak{Q}_{k}^{\text {sep }}$ can be regarded as the disjoint union of $\left\{k^{2}\right\}$ and the set of separable quadratic extensions of $k$.

Definition 3.1. For $x \in V_{k}^{\mathrm{ss}}$, we define

$$
\begin{aligned}
Z_{x} & =\operatorname{Proj} k\left[v_{1}, v_{2}\right] /\left(F_{x}(v)\right), \\
\widetilde{k}(x) & =\Gamma\left(Z_{x}, \mathcal{O}_{Z_{x}}\right) .
\end{aligned}
$$

Also we define $k(x)$ to be the splitting field of $F_{x}(v)$.

Note that $\widetilde{k}(x)$ may not be a field. Since $V_{k}^{\text {ss }}$ is the set of $x$ such that $F_{x}$ does not have a multiple root, $Z_{x}$ is a reduced scheme over $k$ and $\widetilde{k}(x)$ is an element of $\mathfrak{Q}_{k}^{\text {sep }}$ (resp. $\left.\mathfrak{C}_{k}^{\text {sep }}\right)$ for $n=2$ (resp. $\left.n=3\right)$. Since

$$
F_{g x}(v)=\chi_{1}\left(g_{1}\right) F_{x}\left(v g_{2}\right),
$$

the isomorphism classes of $Z_{x}, \widetilde{k}(x)$ and $k(x)$ depend only on the $G_{k}$-orbit of $x$.

We let

$$
\widetilde{\alpha}_{V}: G_{k} \backslash V_{k}^{\mathrm{ss}} \longrightarrow \mathfrak{Q}_{k}^{\mathrm{sep}} \quad\left(\text { resp. } \mathfrak{C}_{k}^{\mathrm{sep}}\right) \quad x \longmapsto \widetilde{k}(x)
$$

for the $D_{4}$ case (resp. the $E_{6}$ case). We first determine the image of $\widetilde{\alpha}_{V}$.

Definition 3.2. (1) For $n=2$, we denote by $\mathfrak{Q}_{k}^{\text {sep }}(D)$ the subset of $\mathfrak{Q}_{k}^{\text {sep }}$ consisting of algebras which have an embedding to $D_{k}$.

(2) For $n=3$, we denote by $\mathfrak{C}_{k}^{\mathrm{sep}}(D)$ the subset of $\mathfrak{C}_{k}^{\mathrm{sep}}$ consisting of algebras which have an embedding to $D_{k}$.

Lemma 3.3. (1) Let $(G, V)$ be the $D_{4}$ case. The image of the map $\widetilde{\alpha}_{V}$ is $\mathfrak{Q}_{k}^{\mathrm{sep}}(D)$.

(2) Let $(G, V)$ be the $E_{6}$ case. The image of the map $\widetilde{\alpha}_{V}$ is $\mathfrak{C}_{k}^{\text {sep }}(D)$.

(3) Moreover, any orbit $G_{k} x \subset V_{k}^{\text {ss }}$ contains an element of the form $y=\left(1, y_{2}\right)$.

Proof. Here we consider the $E_{6}$ case. The $D_{4}$ case can be treated similarly. First note that for $x=(1, w) \in V_{k}^{\mathrm{ss}}, F_{x}\left(v_{1}, 1\right)=\mathcal{N}\left(v_{1}+w\right)$ is the characteristic polynomial of $-w \in D_{k}$ that does not have a multiple root, and hence the algebra $\widetilde{k}(x)$ is isomorphic to the subalgebra $k[w] \subset D_{k}$ generated by $w$ over $k$ in $D_{k}$.

Let $L \in \mathfrak{C}_{k}^{\text {sep }}(D)$. We regard $L$ as a subalgebra of $D_{k}$ and take an element $u \in D_{k}$ so that $L=k[u]$. Let $x=(1,-u) \in V_{k}$. Then since $\operatorname{dim}_{k} L=3=\operatorname{deg} F_{x}\left(v_{1}, 1\right)$, the characteristic polynomial $F_{x}\left(v_{1}, 1\right)$ of $u$ is also the minimum polynomial of $u$. Hence $F_{x}\left(v_{1}, 1\right)$ does not have a multiple root since $u$ is separable. This shows that $x=$ $(1,-u) \in V_{k}^{\text {ss }}$ and now by the remark above we have $\widetilde{\alpha}_{V}\left(G_{k} x\right)=L$. This proves that the image of $\widetilde{\alpha}_{V}$ contains $\mathfrak{C}_{k}^{\mathrm{sep}}(D)$.

Since (3) implies the opposite inclusion, we consider (3). If $D_{k}$ is non-split, this is obvious because $D_{k}$ is a division algebra. We consider the split cases. Since the argument is similar, we consider the $E_{6}$ case here. In this case, $D=\mathrm{M}(3)$ be the algebra of $3 \times 3$ matrices. For $a \in D$, let $\operatorname{rank}(a)$ denote the rank of the matrix $a$. 
Let $x=\left(x_{1}, x_{2}\right) \in V_{k}^{\text {ss }}$. If either the rank of $x_{1}$ or $x_{2}$ is equal to 3 , the element is invertible and hence, there exists a $g \in G_{k}$ such that $g x=(1, *)$. Also if both the rank of $x_{1}$ and $x_{2}$ are less than or equal to 1 , we have $F_{x}(v)=\operatorname{det}\left(x_{1} v_{1}+x_{2} v_{2}\right)=0$ which contradicts to $x \in V_{k}^{\text {ss }}$. Hence, by changing if necessary, we assume that $\operatorname{rank}\left(x_{1}\right)=2$. Then there exists a $g_{1} \in G_{1 k}$ such that $x^{\prime}=g_{1} x=(e, y)$, where

$$
e=\left(\begin{array}{lll}
1 & 0 & 0 \\
0 & 1 & 0 \\
0 & 0 & 0
\end{array}\right), \quad y=\left(\begin{array}{lll}
y_{11} & y_{12} & y_{13} \\
y_{21} & y_{22} & y_{23} \\
y_{31} & y_{32} & y_{33}
\end{array}\right) \text {. }
$$

If $y_{33}=0$, then it is easy to see that $F_{x^{\prime}}(v)$ has a multiple root, and so $y_{33} \neq 0$. Hence, again we can take an element $g_{1}^{\prime} \in G_{1 k}$ so that

$$
g_{1}^{\prime}(e, y)=(e, z), \quad z=\left(\begin{array}{ccc}
z_{11} & z_{12} & 0 \\
z_{21} & z_{22} & 0 \\
0 & 0 & 1
\end{array}\right) \text {. }
$$

Now it is easy to see that there exist $\alpha, \beta \in k$ so that $\operatorname{rank}(\alpha e+\beta z)=3$, hence we have $(3)$.

We later show that the map $\widetilde{\alpha}_{V}$ is in fact injective. Next we consider the structure of the stabilizers for semi-stable points. Note that for $x \in V_{k}^{\mathrm{ss}}$,

$$
\operatorname{dim} G_{x}^{\circ}=\operatorname{dim} G_{x}=\operatorname{dim} G-\operatorname{dim} V=4 .
$$

Lemma 3.4. Let $x \in V_{k}^{\mathrm{ss}}$.

(1) Let $(G, V)$ be the $D_{4}$ case. We have $G_{x}^{\circ} \cong\left(\mathrm{GL}(1)_{\tilde{k}(x)}\right)^{2}$ as a group over $k$.

(2) Let $(G, V)$ be the $E_{6}$ case. We have $G_{x}^{\circ} \cong \mathrm{GL}(1)_{\widetilde{k}(x)} \times \mathrm{GL}(1)_{k}$ as a group over $k$.

Proof. By Lemma $3.3(3)$, any $G_{k}$-orbit in $V_{k}^{\text {ss }}$ contains an element of the form $x=(1, w)$ with $w \in D_{k} \backslash k$. Hence it is enough to show the lemma for these elements. We identify $\widetilde{k}(x)$ with $k[w] \subset D_{k}$.

In order to prove an isomorphism between two algebraic groups $G_{1}, G_{2}$ over $k$, it is enough to construct isomorphisms between the sets $G_{1 R}, G_{2 R}$ of $R$-rational points of $G_{1}, G_{2}$ for all commutative $k$-algebras $R$ which satisfy the usual functorial property. For this, the reader should see [11, p.17].

We first consider (1). For the $D_{4}$ case, $\widetilde{k}(x)=k[w]$ is a separable $k$-algebra of dimension 2 . Let $R$ be any commutative $k$-algebra. We put $\widetilde{R}(x)=\widetilde{k}(x) \otimes R$. Note that $\widetilde{R}(x)=R[w]$ is a subalgebra of $D_{R}=D \otimes R$ and is commutative. Since $\{1, w\}$ is a $k$-basis of $\widetilde{k}(x)$, this is also an $R$-basis of $\widetilde{R}(x)$. Let $s, t \in \widetilde{R}(x)^{\times}$. Then $\{s t, s t w\}$ is also an $R$-basis of $\widetilde{R}(x)$, and so there exists a unique element $g=g_{s t} \in \mathrm{GL}(2)_{R}$ such that $g^{t}(s t, s t w)={ }^{t}(1, w)$. Hence $(s, t) \mapsto\left(s, t, g_{s t}\right)$ gives an injective homomorphism from $\left(\widetilde{R}(x)^{\times}\right)^{2}$ to $G_{x R}$.

This shows that there exists an injective homomorphism

$$
\left(\mathrm{GL}(1)_{\widetilde{k}(x)}\right)^{2} \longrightarrow G_{x}
$$

Since $\left(\mathrm{GL}(1)_{\widetilde{k}(x)}\right)^{2}$ is a connected algebraic group of dimension 4 , we have $\left(\mathrm{GL}(1)_{\widetilde{k}(x)}\right)^{2} \cong$ $G_{x}^{\circ}$

Next we consider (2). Again we let $R$ be any algebra and put $\widetilde{R}(x)=\widetilde{k}(x) \otimes R$. Then we have a injective homomorphism from $\widetilde{R}(x)^{\times} \times R^{\times}$to $G_{x R}$ by sending $(s, t)$ to 
$\left(s, s^{-1} t^{-1}, t\right)$. This shows that there exists an injective homomorphism

$$
\mathrm{GL}(1)_{\tilde{k}(x)} \times \mathrm{GL}(1)_{k} \longrightarrow G_{x}
$$

Since $\mathrm{GL}(1)_{\widetilde{k}(x)} \times \mathrm{GL}(1)_{k}$ is a connected algebraic group of dimension 4 , we have $\mathrm{GL}(1)_{\widetilde{k}(x)} \times$ $\mathrm{GL}(1)_{k} \cong G_{x}^{\circ}$.

Finally, we show the injectivity of $\widetilde{\alpha}_{V}$.

Lemma 3.5. In both cases, the map $\widetilde{\alpha}_{V}$ is injective.

Proof. Since the split case is already proven in [19, we only consider the non-split cases here. Let $x, y \in V_{k}^{\text {ss }}$ satisfy $\widetilde{k}(x) \cong \widetilde{k}(y)$. By Lemma 3.3 (3), we may assume $x=\left(1, u_{1}\right), y=\left(1, u_{2}\right)$. Then $k\left[u_{1}\right]$ and $k\left[u_{2}\right]$ are isomorphic subfields of $D_{k}$. By the Skolem-Noether theorem [1, CHAPITRE $8 \S 10$ ], there exists an element $\theta \in D_{k}^{\times}$such that

$$
k\left[u_{1}\right] \longrightarrow k\left[u_{2}\right], \quad p \longmapsto \theta p \theta^{-1}
$$

gives an isomorphism.

Let $(G, V)$ be the $D_{4}$ case. Then $k\left[u_{1}\right]$ is a quadratic extension over $k$. Hence there exist $a, b \in k$ with $b \neq 0$ such that $u_{2}=\theta\left(a+b u_{1}\right) \theta^{-1}$. Hence for

$$
g=\left(\theta, \theta^{-1},\left(\begin{array}{ll}
1 & 0 \\
a & b
\end{array}\right)\right) \in G_{k},
$$

we have $y=g x$.

Let $(G, V)$ be the $E_{6}$ case. There exists $p \in k\left[u_{1}\right]$ so that $u_{2}=\theta p \theta^{-1}$. We claim that there exist $a, b, c, d \in k$ with $a d-b c \neq 0$ such that $p=\left(c+d u_{1}\right) /\left(a+b u_{1}\right)$. In fact, if we consider the $k$-linear map

$$
\psi: k^{4} \longrightarrow k\left[u_{1}\right], \quad(a, b, c, d) \longmapsto\left(a+b u_{1}\right) p-\left(c+d u_{1}\right),
$$

the kernel of $\psi$ is non-trivial. Therefore there exists $(a, b, c, d) \in k^{4} \backslash\{0\}$ so that $(a+$ $\left.b u_{1}\right) p-\left(c+d u_{1}\right)=0$, and since $p \notin k$, we have $a d-b c \neq 0$.

Hence for

$$
g=\left(\theta\left(a+b u_{1}\right)^{-1}, \theta^{-1},\left(\begin{array}{ll}
a & b \\
c & d
\end{array}\right)\right) \in G_{k},
$$

we have $y=g x$.

We summarize the result in this subsection as follows.

Proposition 3.6. Let $(G, V)$ be the $D_{4}$ case.

(1) The map

$$
G_{k} \backslash V_{k}^{\mathrm{ss}} \longrightarrow \mathfrak{Q}_{k}^{\mathrm{sep}}(D), \quad x \longmapsto \widetilde{k}(x)
$$

is bijective.

(2) Let $x \in V_{k}^{\mathrm{ss}}$. As a group over $k, G_{x}^{\circ} \cong\left(\mathrm{GL}(1)_{\tilde{k}(x)}\right)^{2}$.

Proposition 3.7. Let $(G, V)$ be the $E_{6}$ case.

(1) The map

$$
G_{k} \backslash V_{k}^{\mathrm{ss}} \longrightarrow \mathfrak{C}_{k}^{\mathrm{sep}}(D), \quad x \longmapsto \widetilde{k}(x)
$$

is bijective.

(2) Let $x \in V_{k}^{\mathrm{ss}}$. As a group over $k, G_{x}^{\circ} \cong \mathrm{GL}(1)_{\widetilde{k}(x)} \times \mathrm{GL}(1)_{k}$. 
$\S 3.2$. Application to global fields. If $k$ is a global field, it is well known that the sets $\mathfrak{Q}_{k}^{\text {sep }}(D), \mathfrak{C}_{k}^{\text {sep }}(D)$ can be described by means of local conditions. Here, we review the argument. We assume that $k$ is a global field in this subsection. Also if $D$ is split, $\mathfrak{Q}_{k}^{\text {sep }}(D)=\mathfrak{Q}_{k}^{\text {sep }}$ and $\mathfrak{C}_{k}^{\text {sep }}(D)=\mathfrak{C}_{k}^{\text {sep }}$ for $n=2$ and $n=3$, respectively. Hence we assume $D$ is non-split in this subsection. Recall that $m=n^{2}$ is the dimension of $D$.

Let $\mathfrak{M}$ be the set of places of $k$. For $v \in \mathfrak{M}$, let $k_{v}$ be the completion of $k$ at $v$. We denote by $\operatorname{Inv}_{v}(D)$ the Hasse invariant of $D \otimes k_{v}$ over $k_{v}$.

Definition 3.8. We define $\mathfrak{M}_{D}$ to be the set of elements $v \in \mathfrak{M}$ which $\operatorname{satisfy} \operatorname{Inv}_{v}(D) \neq$ 0 .

It is well known that $\mathfrak{M}_{D}$ is a finite set.

Proposition 3.9. (1) For $n=2$, the set $\mathfrak{Q}_{k}^{\mathrm{sep}}(D)$ consists of elements $L \in \mathfrak{Q}_{k}^{\mathrm{sep}}$ such that $L \otimes k_{v}$ is a quadratic extension of $k_{v}$ for all $v \in \mathfrak{M}_{D}$.

(2) For $n=3$, the set $\mathfrak{C}_{k}^{\text {sep }}(D)$ consists of elements $L \in \mathfrak{C}_{k}^{\text {sep }}$ such that $L \otimes k_{v}$ is a cubic extension of $k_{v}$ for all $v \in \mathfrak{M}_{D}$.

Proof. We will prove the case $n=3$. The case $n=2$ can be treated similarly.

Let $L$ be an arbitrary separable cubic extension of $k$. We denote by $\mathfrak{M}_{L}$ the set of places of $L$. The field $L$ is an element of $\mathfrak{C}_{k}^{\mathrm{sep}}(D)$ if and only if $D$ is split over $L$. By the Hasse principle, this condition is equivalent to that $D \otimes_{k} L_{w} \cong \mathrm{M}(3,3)_{L_{w}}$ for all $w \in \mathfrak{M}_{L}$. Since $D \otimes_{k} k_{v} \cong \mathrm{M}(3,3)_{k_{v}}$ for all $v \notin \mathfrak{M}_{D}$, we only need to consider $w$ which divides an element $v \in \mathfrak{M}_{D}$. For this $v, D_{v}=D \otimes k_{v}$ is a division algebra. Hence for a separable extension $F / k_{v}$ with $\left[F: k_{v}\right] \leq 3, D_{v} \otimes_{k_{v}} F \cong \mathrm{M}(3,3)_{F}$ if and only if $\left[F: k_{v}\right]=3$. Therefore $D_{v} \otimes_{k_{v}} L_{w} \cong \mathrm{M}(3,3)_{L_{w}}$ if and only if $\left[L_{w}: k_{v}\right]=3$.

Remark 3.10. Let $\widetilde{T}=\operatorname{ker}(G \rightarrow \mathrm{GL}(V))$. Then it is easy to that

$$
\widetilde{T}=\left\{\left(t_{11}, t_{12}, t_{2}\right) \mid t_{11}, t_{12}, t_{2} \in \mathrm{GL}(1)_{k}, t_{11} t_{12} t_{2}=1\right\} \cong \mathrm{GL}(1)_{k} \times \mathrm{GL}(1)_{k},
$$

and hence

$$
G_{x}^{\circ} / \widetilde{T} \cong \begin{cases}\left(\mathrm{GL}(1)_{\widetilde{k}(x)} / \mathrm{GL}(1)_{k}\right)^{2} & \text { the } D_{4} \text { case } \\ \mathrm{GL}(1)_{\widetilde{k}(x)} / \mathrm{GL}(1)_{k} & \text { the } E_{6} \text { case. }\end{cases}
$$

For the non-split cases, $\widetilde{k}(x)$ is a quadratic or cubic field over $k$ for any $x \in V_{k}^{\mathrm{ss}}$, and hence $G_{x}^{\circ} / \widetilde{T}$ does not contain a split torus. This shows that $(G, V)$ are of complete type for the non-split cases.

We conclude this subsection with a brief discussion of the possible density theorems which we expect to derive from the theory of the zeta function for our cases.

Roughly speaking, the global zeta function is a counting function for the unnormalized Tamagawa numbers of $G_{x}^{\circ} / \widetilde{T}$ of points in $x \in G_{k} \backslash V_{k}^{\text {ss }}$. Let $F=\widetilde{k}(x)$ and we denote by $h_{F}$ and $R_{F}$ the class number and the regulator of $F$, respectively. If we consider the canonical measure on the adelization of $G_{x}^{\circ} / \widetilde{T}$, the unnormalized Tamagawa number of this group is $\left(\operatorname{Res}_{s=1} \zeta_{F}(s)\right)^{2}\left(\operatorname{resp} . \operatorname{Res}_{s=1} \zeta_{F}(s)\right)$ for the $D_{4}$ case (resp. the $E_{6}$ case) where $\zeta_{F}(s)$ is the Dedekind zeta function. This leads us to believe that the theory of the zeta function will eventually yield the average density of $h_{F}^{2} R_{F}^{2}$ for $F \in \mathfrak{Q}_{k}^{\text {sep }}(D)$ from the $D_{4}$ case, and the average density of $h_{F} R_{F}$ for $F \in \mathfrak{C}_{k}^{\text {sep }}(D)$ from the $E_{6}$ case.

So, we have to develop a local theory to see that these are the correct interpretation of the problem. 


\section{The GLOBAL ZETA FUnCtion}

In this section, we study analytic properties of the global zeta function for non-split cases. The main result is Theorem 4.24, which describe the principal parts of the global zeta function.

$\S$ 4.1. Preliminaries. In this subsection, we collect basic notations that we use in this section. Also, we review some basic facts concerning adelic analysis that we need later. Throughout this section, $k$ is a number field. Let $D$ be a non-split simple algebra of $k$ of dimension 4 or 9 . Then $D$ is a division algebra. Since the argument is similar for the two cases, we treat them simultaneously. Recall that $m=n^{2}$ is the dimension of $D$.

Suppose that $G$ is a locally compact group and $\Gamma$ a discrete subgroup of $G$ contained in the maximal unimodular subgroup of $G$. For any left invariant measure $d g$ on $G$, we choose a left invariant measure $d g$ (we use the same notation, but the meaning will be clear from the context) on $X=G / \Gamma$ so that

$$
\int_{G} f(g) d g=\int_{X} \sum_{\gamma \in \Gamma} f(g \gamma) d g .
$$

Let $r_{1}, r_{2}, h_{k}, R_{k}$ and $\Delta_{k}$ be the number of real places, the number of complex places, the class number, the regulator and the discriminant of $k$, respectively. Let $e_{k}$ be the number of roots of unity contained in $k$. We set

$$
\mathfrak{c}_{k}=2^{r_{1}}(2 \pi)^{r_{2}} h_{k} R_{k} e_{k}^{-1} .
$$

We refer [17] as the basic reference for fundamental properties on adeles. The ring of adeles and the group of ideles are denoted by $\mathbb{A}$ and $\mathbb{A}^{\times}$, respectively. The adelic absolute value || on $\mathbb{A}^{\times}$is normalized so that, for $t \in \mathbb{A}^{\times},|t|$ is the module of multiplication by $t$ with respect to any Haar measure $d x$ on $\mathbb{A}$, i.e. $|t|=d(t x) / d x$. Let $\mathbb{A}^{0}=\left\{t \in \mathbb{A}^{\times}|| t \mid=\right.$ $1\}$. We fix a non-trivial additive character \langle\rangle of $\mathbb{A} / k$. The set of positive real numbers is denoted $\mathbb{R}_{+}$. Suppose $[k: \mathbb{Q}]=n$. For $\lambda \in \mathbb{R}_{+}, \underline{\lambda} \in \mathbb{A}^{\times}$is the idele whose component at any infinite place is $\lambda^{1 / n}$ and whose component at any finite place is 1 . Then we have $|\underline{\lambda}|=\lambda$.

We choose a Haar measure $d x$ on $\mathbb{A}$ so that $\int_{\mathbb{A} / k} d x=1$. We define a Haar measure $d^{\times} t^{0}$ on $\mathbb{A}^{0}$ so that $\int_{\mathbb{A}^{0} / k^{\times}} d^{\times} t^{0}=1$. Using this measure, we choose a Haar measure $d^{\times} t$ on $\mathbb{A}^{\times}$so that

$$
\int_{\mathbb{A}^{\times}} f(t) d^{\times} t=\int_{0}^{\infty} \int_{\mathbb{A}^{0}} f\left(\underline{\lambda} t^{0}\right) d^{\times} \lambda d^{\times} t^{0}
$$

where $d^{\times} \lambda=\lambda^{-1} d \lambda$ and $d \lambda$ is a Lebesgue measure.

Let $\zeta_{k}(s)$ be the Dedekind zeta function of $k$. We define

$$
Z_{k}(s)=\left|\Delta_{k}\right|^{s / 2}\left(\pi^{-s / 2} \Gamma\left(\frac{s}{2}\right)\right)^{r_{1}}\left((2 \pi)^{1-s} \Gamma(s)\right)^{r_{2}} \zeta_{k}(s) .
$$

This definition differs from that in [17, p.129 by the factor of $\left|\Delta_{k}\right|^{s / 2}$ and from that in 20] by the factor of $(2 \pi)^{r_{2}}$. It is adopted here as the most convenient for our purposes. It is well known that $\operatorname{Res}_{s=1} Z_{k}(s)=\mathfrak{c}_{k}$. We define

$$
\phi(s)=\frac{Z_{k}(s)}{Z_{k}(s+1)}, \quad \varrho=\operatorname{Res}_{s=1} \phi(s)=\frac{\mathfrak{c}_{k}}{Z_{k}(2)}, \quad \text { and } \quad \mathfrak{V}_{2}=\varrho^{-1} .
$$

For a complex variable $s$, we denote by $\Re(s)$ the real part. 
For a vector space $V$ over $k, V_{\mathbb{A}}$ denotes its adelization. Let $\mathcal{S}\left(V_{\mathbb{A}}\right)$ be the spaces of Schwartz-Bruhat functions on $V_{\mathbb{A}}$. We define the Haar measure $d x$ on $V_{\mathbb{A}}$ so that $\int_{V_{\mathrm{A}} / V_{k}} d x=1$.

We express elements of $G_{2}=\mathrm{GL}(2)$ as follows:

$$
a\left(t_{1}, t_{2}\right)=\left(\begin{array}{cc}
t_{1} & 0 \\
0 & t_{2}
\end{array}\right), n(u)=\left(\begin{array}{ll}
1 & 0 \\
u & 1
\end{array}\right), \nu=\left(\begin{array}{ll}
0 & 1 \\
1 & 0
\end{array}\right) .
$$

We recall the following well known facts concerning adelic analysis. The proof may be found in [20, Chapter 1].

Lemma 4.1. (1) Let $C \subset \mathrm{GL}(V)_{\mathbb{A}}$ be a compact set, and $\Phi \in \mathcal{S}\left(V_{\mathbb{A}}\right)$. Then there exists $\Psi \in \mathcal{S}\left(V_{\mathbb{A}}\right)$ such that

$$
|\Phi(g x)| \leq \Psi(x)
$$

for all $g \in C, x \in V_{\mathbb{A}}$.

(2) Let $\Phi$ be a Schwartz-Bruhat function on $\mathbb{A}^{n}$. Then there exist Schwartz-Bruhat functions $\Phi_{1}, \ldots, \Phi_{n} \geq 0$ such that

$$
\left|\Phi\left(x_{1}, \ldots, x_{n}\right)\right| \leq \Phi_{1}\left(x_{1}\right) \cdots \Phi_{n}\left(x_{n}\right) .
$$

(3) Suppose $\Phi \in \mathcal{S}(\mathbb{A})$. Then for any $N \geq 1$,

$$
\sum_{x \in k} \Phi(t x) \ll \max \left\{1,|t|^{-1}\right\}, \quad \sum_{x \in k^{\times}} \Phi(t x) \ll|t|^{-N} .
$$

$\S 4.2$. The global zeta function. In this subsection, we define the global zeta function. Also we give an estimate of an incomplete theta series in order to use Shintani's lemma.

Recall that $G_{2}=\mathrm{GL}(2)$. Let $T_{2} \subset G_{2}$ be the set of diagonal matrices and $N_{2} \subset G_{2}$ be the set of lower-triangular matrices whose diagonal entries are 1 . Then $B_{2}=T_{2} N_{2}$ is a Borel subgroup of $G_{2}$.

Let

$$
\begin{aligned}
G_{1 \mathbb{A}}^{0} & =\left\{g_{1}=\left(g_{11}, g_{12}\right) \in G_{1 \mathbb{A}}|| \mathcal{N}\left(g_{11}\right)|=| \mathcal{N}\left(g_{12}\right) \mid=1\right\}, \\
G_{2 \mathbb{A}}^{0} & =\left\{g_{2} \in G_{2 \mathbb{A}}|| \operatorname{det} g_{2} \mid=1\right\}, \\
G_{\mathbb{A}}^{0} & =G_{1 \mathbb{A}}^{0} \times G_{2 \mathbb{A}}^{0}, \quad \bar{G}_{\mathbb{A}}=\mathbb{R}_{+} \times G_{\mathbb{A}}^{0}, \\
\widehat{T}_{2 \mathbb{A}}^{0} & =\left\{a\left(t_{21}, t_{22}\right) \mid t_{21}, t_{22} \in \mathbb{A}^{0}\right\}, \\
T_{2 \mathbb{A}}^{0} & =\left\{a\left(\underline{\mu}^{-1}, \underline{\mu}\right) t_{2} \mid \mu \in \mathbb{R}_{+}, t_{2} \in \widehat{T}_{2 \mathbb{A}}^{0}\right\}, \\
B_{2 \mathbb{A}}^{0} & =T_{2 \mathbb{A}}^{0} N_{2 \mathbb{A}}, \quad P_{\mathbb{A}}^{0}=G_{1 \mathbb{A}}^{0} \times B_{2 \mathbb{A}}^{0} .
\end{aligned}
$$

The group $\bar{G}_{\mathbb{A}}$ acts on $V_{\mathbb{A}}$ by assuming that $\lambda \in \mathbb{R}_{+}$acts by multiplication by $\underline{\lambda}$. Throughout this section, we express elements $\bar{g} \in \bar{G}_{\mathbb{A}}, g^{0} \in G_{\mathbb{A}}^{0}$ as

$$
\bar{g}=\left(\lambda, g_{1}, g_{2}\right), g^{0}=\left(g_{1}, g_{2}\right)
$$

where $\lambda \in \mathbb{R}_{+}, g_{1} \in G_{1 \mathbb{A}}^{0}$, and $g_{2} \in G_{2 \mathbb{A}}^{0}$. We identify element $g^{0} \in G_{\mathbb{A}}^{0}$ with $\left(1, g^{0}\right) \in \bar{G}_{\mathbb{A}}$ and $g_{1} \in G_{1 \mathbb{A}}^{0}, g_{2} \in G_{2 \mathbb{A}}^{0}$ with $\left(1, g_{1}, 1\right),\left(1,1, g_{2}\right)$. We may also write as $\bar{g}=\underline{\lambda} g^{0}$.

Let $K_{2}$ be the standard maximal compact subgroup of $G_{2 \mathbb{A}}^{0}$ i.e.

$$
K_{2}=\prod_{v \in \mathfrak{M}_{\mathbb{R}}} \mathrm{O}(2, \mathbb{R}) \times \prod_{v \in \mathfrak{M}_{\mathbb{C}}} \mathrm{U}(2, \mathbb{C}) \times \prod_{v \in \mathfrak{M}_{\mathfrak{f}}} \mathrm{GL}\left(2, \mathcal{O}_{v}\right)
$$

Let $d \kappa_{2}$ be the Haar measure on $K_{2}$ such that the total volume of $K_{2}$ is 1 . 
Let

$$
t_{2}=a_{2}\left(\underline{\mu}^{-1} t_{21}, \underline{\mu} t_{22}\right), b_{2}=t_{2} n_{2}(u)
$$

where $\mu \in \mathbb{R}_{+}, t_{21}, t_{22} \in \mathbb{A}^{0}, u \in \mathbb{A}$. Throughout this section, we assume that

$$
g_{2}=\kappa_{2} b_{2}=\kappa_{2} a_{2}\left(\underline{\mu}^{-1} t_{21}, \underline{\mu} t_{22}\right) n_{2}(u)
$$

is the Iwasawa decomposition of $g_{2} \in G_{2 \mathbb{A}}^{0}$.

The measure $d u$ on $\mathbb{A}$ induces an invariant measure on $N_{\mathbb{A}}$. We put

$$
d^{\times} t=d^{\times} \mu d^{\times} t_{21} d^{\times} t_{22}, d b_{2}=\mu^{2} d^{\times} t_{2} d u_{2}
$$

We use $d g_{2}=d \kappa_{2} d b_{2}^{0}$ as the Haar measure on $G_{2 \mathbb{A}}^{0}$. It is well known that the volume of $G_{2 \mathbb{A}}^{0} / G_{2 k}$ with respect to the measure $d g_{2}$ is $\mathfrak{V}_{2}$.

We fix an arbitrary Haar measure $d g_{1}$ on $G_{1 \mathbb{A}}^{0}$. Since the rank of the group $G_{1}$ is 0 , $G_{1 \mathbb{A}}^{0} / G_{1 k}$ is compact. We put

$$
\tau\left(G_{1}\right)=\int_{G_{1 \AA}^{0} / G_{1 k}} d g_{1} .
$$

We choose $d g^{0}=d g_{1} d g_{2}, d \bar{g}=d^{\times} \lambda d g^{0}$ as Haar measures on $G_{\mathbb{A}}^{0}, \bar{G}_{\mathbb{A}}$, respectively.

For $\eta>0$, we define

$$
T_{2 \eta+}^{0}=\left\{a\left(\underline{\mu}^{-1}, \underline{\mu}\right) \mid \mu \in \mathbb{R}_{+}, \mu \leq \eta\right\} .
$$

Let $\Omega_{2} \subset \widehat{T}_{2 \mathbb{A}}^{0} N_{2 \mathbb{A}}$ be a compact subset. We define $\mathfrak{S}_{2}^{0}=K_{2} T_{2 \eta+}^{0} \Omega_{2}$. It is well known that for a suitable choice of $\eta$ and $\Omega_{2}, \mathfrak{S}_{2}^{0}$ surjects to $G_{2 \mathbb{A}}^{0} / G_{2 k}$. Also there exists another compact set $\widehat{\Omega}_{2} \in G_{2 \mathbb{A}}^{0}$ such that $\mathfrak{S}_{2}^{0} \subset \widehat{\Omega}_{2} T_{2 \eta+}^{0}$. We fix a compact subset $\widehat{\Omega}_{1} \subset G_{1 \mathbb{A}}^{0}$ which surjects to $G_{1 \mathbb{A}}^{0} / G_{1 k}$. Let $\widehat{\Omega}=\widehat{\Omega}_{1} \times \widehat{\Omega}_{2}$.

Definition 4.2. Let $r \in \mathbb{R}$. We define $C\left(G_{\AA}^{0} / G_{k}, r\right)$ to be the set of continuous functions $f\left(g^{0}\right)$ on $G_{\AA}^{0} / G_{k}$ satisfying

$$
\sup _{g^{0} \in \widehat{\Omega} T_{2 \eta+}^{0}} f\left(g^{0}\right) \mu^{-r}<\infty .
$$

A function $f$ on $G_{\mathbb{A}}^{0} / G_{k}$ is said to be slowly increasing if $f \in C\left(G_{\mathbb{A}}^{0} / G_{k}, r\right)$ for some $r \in \mathbb{R}$.

Note that $C\left(G_{\mathbb{A}}^{0} / G_{k}, r_{1}\right) \subset C\left(G_{\mathbb{A}}^{0} / G_{k}, r_{2}\right)$ if $r_{1}>r_{2}$ and $C\left(G_{\mathbb{A}}^{0} / G_{k}, r\right) \subset L^{1}\left(G_{\mathbb{A}}^{0} / G_{k}, d g^{0}\right)$ if $r>-2$.

Definition 4.3. Let $\Phi \in \mathcal{S}\left(V_{\mathbb{A}}\right)$ and $\bar{g} \in \bar{G}_{\mathbb{A}}$. For any subset $L \subset V_{k}$, we define an incomplete theta series $\Theta_{L}(\Phi, \bar{g})$ by

$$
\Theta_{L}(\Phi, \bar{g})=\sum_{x \in L} \Phi(\bar{g} x)
$$

If $L$ is invariant under the action of a subgroup $H_{k} \subseteq G_{k}$, we often regard $\Theta_{L}(\Phi, \bar{g})$ as a function on $\bar{G}_{\mathbb{A}} / H_{k}$. For example, if $L=V_{k}^{\mathrm{ss}}, \Theta_{V_{k}^{\mathrm{ss}}}(\Phi, \bar{g})$ is a function on $\bar{G}_{\mathbb{A}} / G_{k}$.

Lemma 4.4. For any $N \geq 1$,

$$
\Theta_{V_{k}^{\mathrm{ss}}}(\Phi, \bar{g}) \ll \begin{cases}\lambda^{-2 N-2} \mu^{-1} & \lambda \geq 1, \\ \lambda^{-2-m} \mu^{-1} & \lambda \leq 1,\end{cases}
$$

for $\bar{g} \in \mathbb{R}_{+} \times \mathfrak{S}^{0}$. 
Proof. By (1) of Lemma 4.1, we may assume $\bar{g}=\underline{\lambda}\left(1, a_{2}\left(\mu^{-1}, \mu\right)\right), \mu \ll 1$. For $x=$ $\left(x_{1}, x_{2}\right) \in V_{k}^{\text {ss }}$, we have $x_{1} \neq 0$ and $x_{2} \neq 0$. Note that the weight of $a\left(t_{1}, t_{2}\right) \in T_{2}$ with respect to each $k$-coordinate of $x_{1}$ and $x_{2}$ is $t_{1}$ and $t_{2}$, respectively. Hence, by (2) and (3) of Lemma 4.1, for any $N_{1}, N_{2} \geq 1$,

$$
\begin{aligned}
\Theta_{V_{k}^{\text {ss }}}(\Phi, \bar{g}) & \ll\left(\lambda^{-1} \mu\right)^{N_{1}}\left(\lambda^{-1} \mu^{-1}\right)^{N_{2}} \max \left(1, \lambda^{-1} \mu\right)^{m-1} \max \left(1, \lambda^{-1} \mu^{-1}\right)^{m-1} \\
& \leq \lambda^{-N_{1}-N_{2}} \mu^{N_{1}-N_{2}} \max \left(1, \lambda^{2-2 m}\right) \max \left(1, \mu^{m-1}\right) \max \left(1, \mu^{1-m}\right) \\
& \ll \lambda^{-N_{1}-N_{2}} \max \left(1, \lambda^{2-2 m}\right) \mu^{N_{1}-N_{2}+1-m} .
\end{aligned}
$$

Note that $\max \{1, a b\} \leq \max \{1, a\} \cdot \max \{1, b\}$ for $a, b \geq 0$. For $\lambda \geq 1$, take $N_{1}=$ $N+m-2, N_{2}=N$. For $\lambda \leq 1$, take $N_{1}=3, N_{2}=1$. Then we have the proposition.

Now we define the global zeta function.

Definition 4.5. For $\Psi \in \mathcal{S}\left(V_{\mathbb{A}}\right)$ and a complex variable $s$, we define

$$
\begin{aligned}
Z(\Phi, s) & =\int_{\bar{G}_{\mathbb{A}} / G_{k}} \lambda^{s} \Theta_{V_{k}^{\mathrm{ss}}}(\Phi, \bar{g}) d \bar{g}, \\
Z_{+}(\Phi, s) & =\int_{\substack{\bar{G}_{\mathbb{A}} / G_{k} \\
\lambda \geq 1}} \lambda^{s} \Theta_{V_{k}^{\mathrm{ss}}}(\Phi, \bar{g}) d \bar{g} .
\end{aligned}
$$

The integral $Z(\Phi, s)$ is called the global zeta function. By Lemma 4.4 the integral $Z(\Phi, s)$ converges absolutely and locally uniformly on a certain right half-plane and the integral $Z_{+}(\Phi, s)$ is an entire function. Since the global zeta function is well defined for $V_{k}^{\text {ss }}$, by Theorem (0.3.7) in [20] (which is due to Shintani), $Z(\Phi, s)$ can be continued meromorphically to the entire plane and satisfies a functional equation

$$
Z(\Phi, s)=Z(\widehat{\Phi}, 2 m-s)
$$

The purpose of this section is to determine the pole structure and to describe the residues by means of certain distributions.

Remark 4.6. The above definition of the zeta function looks slightly different from the original definition in [15], but these are essentially the same. We briefly compare these functions. Let $\widetilde{G}=G / \widetilde{T}$. Recall that we put $\widetilde{T}=\operatorname{ker}(G \rightarrow \operatorname{GL}(V))$. Let $d \widetilde{g}$ be an invariant measure on $\widetilde{G}_{\mathbb{A}}$. The original definition of the global zeta function is as follows:

$$
Z^{*}(\Phi, s)=\int_{\widetilde{G}_{\mathbb{A}} / \widetilde{G}_{k}}|\chi(\widetilde{g})|^{s} \Theta_{V_{k}^{\mathrm{ss}}}(\Phi, \widetilde{g}) d \widetilde{g}
$$

Since $\widetilde{T} \cong \mathrm{GL}(1) \times \mathrm{GL}(1)$ is a split torus, the first Galois cohomology set $H^{1}\left(k^{\prime}, \widetilde{T}\right)$ is trivial for any field $k^{\prime}$ containing $k$. This implies that the set of $k^{\prime}$-rational point of $\widetilde{G}$ coincides with $G_{k^{\prime}} / \widetilde{T}_{k^{\prime}}$. Therefore $\widetilde{G}_{\mathbb{A}}=G_{\mathbb{A}} / \widetilde{T}_{\mathbb{A}}$ and $\widetilde{G}_{\mathbb{A}} / \widetilde{G}_{k}=G_{\mathbb{A}} / \widetilde{T}_{\mathbb{A}} G_{k}$. Let $\widetilde{T}_{\mathbb{A}}^{0}=G_{\mathbb{A}}^{0} \cap \widetilde{T}_{\mathbb{A}}$. Then we have

$$
\left(\mathbb{R}_{+} \times G_{\mathbb{A}}^{0}\right) / \widetilde{T}_{\mathbb{A}}^{0} \cong G_{\mathbb{A}} / \widetilde{T}_{\mathbb{A}}
$$

via the map which sends the class of $\left(\lambda, g_{11}, g_{12}, g_{2}\right)$ to class of $\left(g_{11}, g_{12}, \underline{\lambda} g_{2}\right)$. Moreover, this map is compatible with their actions on $V_{\mathbb{A}}$. If we identify $\bar{G}_{\mathbb{A}} / \widetilde{T}_{\mathbb{A}}^{0}$ with $\widetilde{G}_{\mathbb{A}}$ via the isomorphism, then we have $|\chi(\bar{g})|=\lambda^{4}$ for the $D_{4}$ case and $|\chi(\bar{g})|=\lambda^{12}$ for the $E_{6}$ case. Also the volume of $\widetilde{T}_{\mathbb{A}}^{0} / \widetilde{T}_{k} \cong\left(\mathbb{A}^{0} / k^{\times}\right)^{2}$ is finite. Hence $Z(\Phi, 4 s)$ is a constant multiple of $Z^{*}(\Phi, s)$ for the $D_{4}$ case and $Z(\Phi, 12 s)$ is a constant multiple of $Z^{*}(\Phi, s)$ for the $E_{6}$ 
case. (The constant depends on the choice of the measure.) Our choice of $Z(\Phi, s)$ is for the conventions of our global theory.

Let

$$
\mathrm{M} \Phi(x)=\int_{K_{2}} \Phi\left(\kappa_{2} x\right) d \kappa_{2}
$$

Then $Z(\Phi, s)=Z(\mathrm{M} \Phi, s)$ and $\mathrm{M} \Phi(x)$ is $K_{2}$-invariant. Therefore, we may assume the following for the rest of this section.

Assumption 4.7. The Schwartz-Bruhat function $\Phi$ is $K_{2}$-invariant.

$\S 4.3$. The principal part. For $x=\left(x_{1}, x_{2}\right)$ and $y=\left(y_{1}, y_{2}\right)$, we define

$$
[x, y]=\mathcal{T}\left(x_{1} y_{2}\right)+\mathcal{T}\left(x_{2} y_{1}\right)
$$

This is a non-degenerate bilinear form on $V$. For $\bar{g}=\left(\lambda, g_{11}, g_{12}, g_{2}\right) \in \mathbb{R}_{+} \times G_{\mathbb{A}}^{0}$, we define

$$
\bar{g}^{\iota}=\left(\lambda^{-1}, g_{12}^{-1}, g_{11}^{-1}, \nu^{t} g_{2}^{-1} \nu\right) .
$$

This is an involution and the above bilinear form satisfies

$$
\left[\bar{g} x, \bar{g}^{\iota} y\right]=[x, y] \text {. }
$$

Recall that \langle\rangle is a non-trivial additive character of $\mathbb{A} / k$. For $\Phi \in \mathcal{S}\left(V_{\mathbb{A}}\right)$, we define its Fourier transform by

$$
\widehat{\Phi}(x)=\int_{V_{\mathbb{A}}} \Phi(y)\langle[x, y]\rangle d y .
$$

It is easy to see that the Fourier transform of $\Phi(\bar{g} \cdot)$ is $\lambda^{-8} \widehat{\Phi}\left(\bar{g}^{\iota} \cdot\right)$.

For $\lambda \in \mathbb{R}_{+}$, we define $\Phi_{\lambda}(x)=\Phi(\underline{\lambda} x)$.

Definition 4.8. For $\Phi \in \mathcal{S}\left(V_{\mathbb{A}}\right), s \in \mathbb{C}$ and $g^{0} \in G_{\mathbb{A}}^{0}$, we define

$$
\begin{aligned}
J\left(\Phi, g^{0}\right) & =\sum_{x \in S_{k}} \widehat{\Phi}\left(\left(g^{0}\right)^{\iota} x\right)-\sum_{x \in S_{k}} \Phi\left(g^{0} x\right), \\
I^{0}(\Phi) & =\int_{G_{\AA}^{0} / G_{k}} J\left(\Phi, g^{0}\right) d g^{0}, \\
I(\Phi, s) & =\int_{0}^{1} \lambda^{s} I^{0}\left(\Phi_{\lambda}\right) d^{\times} \lambda .
\end{aligned}
$$

Then by the Poisson summation formula, we have the following.

Proposition 4.9. We have

$$
Z(\Phi, s)=Z_{+}(\Phi, s)+Z_{+}(\widehat{\Phi}, 2 m-s)+I(\Phi, s) .
$$

We study the last term for the rest of this section.

$\S$ 4.4. Stratification. In this subsection, we consider a stratification of $V_{k}$. Let

$$
Y_{1}=\left\{x \in V \mid x_{1}=0\right\}, \quad Y_{1}^{\mathrm{ss}}=\left\{x \in Y_{1} \mid x_{2} \neq 0\right\} .
$$

We define $S_{1}=G Y_{1}^{\mathrm{ss}}$. Let $P=G_{1} \times B_{2}$.

Lemma 4.10. We have

(1) $V_{k} \backslash\{0\}=V_{k}^{\text {ss }} \amalg S_{1 k}$,

(2) $S_{1 k}=G_{k} \times_{P_{k}} Y_{1 k}^{\mathrm{ss}}$. 
Proof. We consider (1). Let $x \in V_{k} \backslash\{0\}$ and $x \notin V_{k}^{\text {ss }}$. Since either $x_{1} \neq 0$ or $x_{2} \neq 0$, there exists an element $g \in G_{k}$ such that the first coordinate of $g x$ is 1 . Replacing $x$ by $g x$, we may assume that $x$ is of the form $x=\left(1,-x_{2}\right)$, where $x_{2} \in W_{k}$. Then $F_{x}(v)$ is the characteristic polynomial of $x_{2}$ and the condition $P(x)=0$ is equivalent to that the characteristic polynomial of $x_{2}$ has a multiple root.

Let $L=k\left[x_{2}\right]$ be the subalgebra of $D_{k}$ generated by $x_{2}$ over $k$. Since $D_{k}$ has no zero divisor, $k\left[x_{2}\right]$ is a (commutative) integral domain which is finite over the field $k$. So it is a field. Then since the degree of extension $[L: k] \operatorname{divides} \operatorname{dim}_{k} D_{k}=n^{2}$, it is either 1 or $n$. Note that we are assuming $n=2$ or 3 . Assume $[L: k]=n$. Then $F_{x}(v)$ is a minimum polynomial of $x_{2}$ over $k$ because the degree of $F_{x}(v)$ is $n$. Since any field extension of an algebraic number field is separable, we conclude that $F_{x}(v)$ does not have a multiple root. This is an contradiction and hence $[L: k]=1$, which implies $x_{2} \in k$. Therefore, there exists an element $g_{2} \in G_{2 k}$ such that $g_{2} x \in Y_{1 k}^{\mathrm{ss}}$. This proves (1).

It is easy to see that $P_{1 k} Y_{1 k}^{\mathrm{ss}}=Y_{1 k}^{\mathrm{ss}}$ and that if $x \in Y_{1 k}^{\mathrm{ss}}, g \in G_{k}$ and $g x \in Y_{1 k}^{\mathrm{ss}}$ then $g \in P_{k}$. This proves $(2)$.

$\S 4.5$. The smoothed Eisenstein series. To compute $I^{0}(\Phi)$, it seems natural to divide the index set $S_{k}$ of the summation into its $G_{k}$-orbits and perform integration separately. However, we can not put this into practice because the corresponding integrals diverge. This is the main difficulty when one calculates the global zeta functions of the prehomogeneous vector spaces. To surmount this problem Shintani [16] introduced the smoothed Eisenstein series of GL(2). He used this series to determine the principal parts of the global zeta functions for the space of binary cubic forms and the space of binary quadratic forms. Later A. Yukie [20] generalized the theory of Eisenstein series to the products of GL( $n)$ 's, and applied it to determine the principal parts of the global zeta functions in some cases. In this subsection, we essentially repeat the argument of Shintani and Yukie in our settings.

We express the Iwasawa decomposition of $g_{2} \in G_{2 \mathbb{A}}^{0}$ as

$$
g_{2}=\kappa_{2}\left(g_{2}\right) a_{2}\left(t_{21}\left(g_{2}\right), t_{22}\left(g_{2}\right)\right) n_{2}\left(u\left(g_{2}\right)\right) .
$$

Let $s \in \mathbb{C}$. The Eisenstein series of $G_{2 \mathbb{A}}^{0}$ for $B_{2}$ is defined as

$$
E\left(g_{2}, s\right)=\sum_{\gamma \in G_{2 k} / B_{2 k}}\left|t_{21}\left(g_{2} \gamma\right)\right|^{s+1}
$$

It is well known that the summation defining $E\left(g_{2}, s\right)$ converges absolutely and locally uniformly in certain right half-plane and can be continued meromorphically to the whole complex plane. For analytic properties of $E\left(g_{2}, s\right)$, see [18], 20].

Let $\psi(s)$ be an entire function of $s$ such that

$$
\sup _{c_{1}<\Re(s)<c_{2}}\left(1+|s|^{N}\right)|\psi(s)|<\infty
$$

for all $c_{1}<c_{2}, N>0$. Moreover, we assume $\psi(1) \neq 0$. For a complex variable $w$, we define

$$
\Lambda_{\psi}(w ; s)=\frac{\psi(s)}{w-s} .
$$

Where there is no confusion, we drop $\psi$ and use the notation $\Lambda(w ; s)$ instead. 
Definition 4.11. For a complex variable $w$, we define

$$
\mathcal{E}\left(g^{0}, w, \psi\right)=\frac{1}{2 \pi \sqrt{-1}} \int_{\Re(s)=r_{1}} E\left(g_{2}, s\right) \Lambda_{\psi}(w ; s) d s .
$$

for some $r_{1}>1$.

Note that the above definition does not depend on the choice of $r_{1}$. The function $\mathcal{E}\left(g^{0}, w, \psi\right)$ is called the smoothed Eisenstein series. When there is no confusion, we drop $\psi$ and use the notation $\mathcal{E}\left(g^{0}, w\right)$ instead.

The following proposition is known as Shintani's lemma.

Proposition 4.12. (1) The function $\mathcal{E}\left(g^{0}, w\right)$ is holomorphic for $\Re(w)>0$ except for a simple pole at $w=1$ with the residue $\varrho \psi(1)$.

(2) Let $f \in C\left(G_{\mathbb{A}}^{0} / G_{k}, r\right)$ for some $r>-2$. Then the integral

$$
\int_{G_{\AA}^{0} / G_{k}} f\left(g^{0}\right) \mathcal{E}\left(g^{0}, w\right) d g^{0}
$$

becomes a holomorphic function for $\Re(w)>1-\epsilon$ for a constant $\epsilon>0$ except possibly for a simple pole at $w=1$ with residue

$$
\varrho \psi(1) \int_{G_{\AA}^{0} / G_{k}} f\left(g^{0}\right) d g^{0} .
$$

(3) For a slowly increasing function $f\left(g^{0}\right)$ on $G_{\mathbb{A}}^{0} / G_{k}$, the integral

$$
\int_{G_{\AA}^{0} / G_{k}} f\left(g^{0}\right) \mathcal{E}\left(g^{0}, w\right) d g^{0}
$$

becomes a holomorphic function on a certain right half-plane.

(4) We have

$$
\int_{G_{\AA}^{0} / G_{k}} \mathcal{E}\left(g^{0}, w\right) d g^{0}=\tau\left(G_{1}\right) \Lambda(w ; 1) .
$$

The above proposition was first proved for GL(2) by Shintani [16, pp. 172, 173, 177]. The adelic proof is given in [18, pp. 527, 528]. In our case, we included the first factor $G_{1 \mathbb{A}}^{0}$ in the statement instead of just considering GL(2), but exactly the same proof works because $G_{1 \mathbb{A}}^{0} / G_{1 k}$ is compact. For the convenience of the reader, we give a sketch of the proof here. To prove Proposition 4.12, the following lemma on the Eisenstein series for $G_{2}=\mathrm{GL}(2)$ is crucial. For the proof, see [16], [18], or [20].

Lemma 4.13. (1) Let $E_{N_{2}}\left(g_{2}, s\right)$ be the constant term of $E\left(g_{2}, w\right)$ with respect to $N_{2}$ i.e.

$$
E_{N_{2}}\left(g_{2}, s\right)=\int_{N_{2 \mathbb{A}} / N_{2 k}} E\left(g_{2} n_{2}(u), s\right) d u .
$$

The constant term has the following explicit formula:

$$
E_{N_{2}}\left(g_{2}, s\right)=\mu^{-s-1}+\mu^{s-1} \phi(s) .
$$

(2) Let $\widetilde{E}\left(g_{2}, s\right)=E\left(g_{2}, s\right)-E_{N_{2}}\left(g_{2}, s\right)$ be the non-constant term. Then $\widetilde{E}\left(g_{2}, s\right)$ is holomorphic for $\Re(s)>0$. Moreover, for any $s$ in this region and $l>1$,

$$
\left|\widetilde{E}\left(g_{2}, s\right)\right| \ll \mu^{2 l-1} .
$$


(3) We have

$$
\int_{G_{2 \mathbb{A}}^{0} / G_{2 k}} \mathcal{E}\left(g_{2}, w\right) d g_{2}=\Lambda(w, 1) .
$$

Sketch of the proof of Proposition 4.12. Let

$$
E^{\prime}\left(g_{2}, s\right)=E\left(g_{2}, s\right)-\mu^{s-1} \phi(s)=\mu^{-s-1}+\widetilde{E}\left(g_{2}, s\right) .
$$

This function is holomorphic for $\Re(s)>0$. Recall that $\rho=\operatorname{Res}_{s=1} \phi(s)$.

Let $\epsilon$ be a small positive number. By moving the contour, we have

$$
\begin{aligned}
& \frac{1}{2 \pi \sqrt{-1}} \int_{\Re(s)=r_{1}} \mu^{s-1} \phi(s) \frac{\psi(s)}{w-s} d s=\frac{\rho \psi(1)}{w-1}+\frac{1}{2 \pi \sqrt{-1}} \int_{\Re(s)=\epsilon} \mu^{s-1} \phi(s) \frac{\psi(s)}{w-s} d s, \\
& \frac{1}{2 \pi \sqrt{-1}} \int_{\Re(s)=r_{1}} E^{\prime}\left(g_{2}, s\right) \frac{\psi(s)}{w-s} d s=\frac{1}{2 \pi \sqrt{-1}} \int_{\Re(s)=\epsilon} E^{\prime}\left(g_{2}, s\right) \frac{\psi(s)}{w-s} d s,
\end{aligned}
$$

and each of the integrals in the right hand sides is holomorphic for $\Re(w)>\epsilon$. This proves (1).

We consider (2). Since $C\left(G_{\AA}^{0} / G_{k}, r_{1}\right) \subset C\left(G_{\AA}^{0} / G_{k}, r_{2}\right)$ for $r_{1}>r_{2}$, we may assume $-2<r<0$. By moving the contour, we have

$$
\begin{aligned}
& \frac{1}{2 \pi \sqrt{-1}} \int_{\Re(s)=r_{1}}\left(\int_{G_{\AA}^{0} / G_{k}} f\left(g^{0}\right) \mu^{s-1} d g^{0}\right) \phi(s) \frac{\psi(s)}{w-s} d s \\
& =\frac{\rho \psi(1)}{w-1} \int_{G_{\AA}^{0} / G_{k}} f\left(g^{0}\right) d g^{0} \\
& \quad+\frac{1}{2 \pi \sqrt{-1}} \int_{\Re(s)=-r / 2}\left(\int_{G_{\AA}^{0} / G_{k}} f\left(g^{0}\right) \mu^{s-1} d g^{0}\right) \phi(s) \frac{\psi(s)}{w-s} d s .
\end{aligned}
$$

The second term in the right hand side is holomorphic if $\Re(w)>-r / 2$ because for $\Re(s)=-r / 2$, we have $f\left(g^{0}\right) \mu^{s-1} \in C\left(G_{\AA}^{0} / G_{k},(r-2) / 2\right) \subset L^{1}\left(G_{\AA}^{0} / G_{k}, d g^{0}\right)$. A similar argument shows that

$$
\frac{1}{2 \pi \sqrt{-1}} \int_{\Re(s)=r_{1}}\left(\int_{G_{\AA}^{0} / G_{k}} f\left(g^{0}\right) E^{\prime}\left(g_{2}, s\right) d g^{0}\right) \frac{\psi(s)}{w-s} d s
$$

is holomorphic for $\Re(w)>0$. This proves $(2)$.

To see (3), we may assume $f\left(g^{0}\right) \in C\left(G_{\AA}^{0} / G_{k}, r\right)$ for some $r \leq-2$. Then $f\left(g^{0}\right) \mu^{s-1} \in$ $C\left(G_{\mathbb{A}}^{0} / G_{k},-1\right)$ for $\Re(s) \geq-r, f\left(g^{0}\right) \mu^{-s-1} \in C\left(G_{\mathbb{A}}^{0} / G_{k},-1\right)$ for $\Re(s) \leq r$, and $f\left(g^{0}\right) \widetilde{E}\left(g_{2}, s\right) \in$ $C\left(G_{\AA}^{0} / G_{k},-1\right)$ for $\Re(s)>\epsilon$ where $\epsilon>0$ is a small number. Hence by moving the contour for each of these terms, we obtain (3). Now (4) immediately follows from Lemma 4.13 (3), and these finish the proof of Proposition 4.12,

Let $\mathcal{E}_{N_{2}}\left(g^{0}, w\right)$ be the constant term of $\mathcal{E}\left(g^{0}, w\right)$ with respect to $N_{2}$ i.e.

$$
\mathcal{E}_{N_{2}}\left(g^{0}, w\right)=\int_{N_{2 \mathbb{A}} / N_{2 k}} \mathcal{E}\left(g^{0} n(u), w\right) d u .
$$

By Lemma 4.13 (1), we have

$$
\mathcal{E}_{N_{2}}\left(g^{0}, w\right)=\frac{1}{2 \pi \sqrt{-1}} \int_{\Re(s)=r_{1}}\left(\mu^{-s-1}+\mu^{s-1} \phi(s)\right) \Lambda(w ; s) d s .
$$


Definition 4.14. Let $f(w), g(w)$ be holomorphic functions of $w \in \mathbb{C}$ in some right half-plane. We use the notation $f(w) \sim g(w)$ if $f(w)-g(w)$ can be continued meromorphically to $\{w \mid \Re(w)>1-\epsilon\}$ for some $\epsilon>0$ and is holomorphic at $w=1$.

We define

$$
I^{0}(\Phi, w)=\int_{G_{\AA}^{0} / G_{k}} J\left(\Phi, g^{0}\right) \mathcal{E}\left(g^{0}, w\right) d g^{0} .
$$

By Lemma 4.4 $J(\Phi, g) \in C\left(G_{\mathbb{A}}^{0} / G_{k},-1\right)$. Hence, by Proposition 4.12 (2), we have the following.

Proposition 4.15. We have

$$
I^{0}(\Phi, w) \sim \varrho \Lambda(w ; 1) I^{0}(\Phi) .
$$

Definition 4.16. For a complex variable $w$, we define

$$
\begin{aligned}
& \Xi_{1}(\Phi, w)=\int_{G_{\AA}^{0} / G_{k}} \Theta_{S_{1 k}}\left(\Phi, g^{0}\right) \mathcal{E}\left(g^{0}, w\right) d g^{0}, \\
& \Xi_{\#}(\Phi, w)=\Phi(0) \int_{G_{\AA}^{0} / G_{k}} \mathcal{E}\left(g^{0}, w\right) d g^{0} .
\end{aligned}
$$

Since $\Theta_{S_{k}^{\prime}}\left(\Phi, g^{0}\right)$ is a slowly increasing function, by Proposition 4.12 (3), the integral $\Xi_{1}(\Phi, w)$ converges absolutely for sufficiently large $\Re(w)$. It is proved in 20] that $E\left(g_{2}, s\right)=E\left({ }^{t} g_{2}^{-1}, s\right)$ for $g_{2} \in G_{2 \mathbb{A}}^{0}$. Hence, $\mathcal{E}\left(g^{0}, w\right)=\mathcal{E}\left(\left(g^{0}\right)^{\iota}, w\right)$ for $g^{0} \in G_{\mathbb{A}}^{0}$. Therefore, by Lemma 4.10, we have the following.

Proposition 4.17. We have

$$
I^{0}(\Phi, w)=\Xi_{1}(\widehat{\Phi}, w)+\Xi_{\#}(\widehat{\Phi}, w)-\Xi_{1}(\Phi, w)-\Xi_{\#}(\Phi, w) .
$$

For $\Xi_{\#}(\Phi, w)$, Proposition 4.12 immediately leads to the following.

Proposition 4.18. We have

$$
\Xi_{\#}(\Phi, w)=\Phi(0) \tau\left(G_{1}\right) \mathfrak{V}_{2} \varrho \Lambda(w, \rho) .
$$

We study $\Xi_{1}(\Phi, w)$ in $\$ 4.7$

$\S 4.6$. The zeta function associated with the space of division algebra. Since the prehomogeneous vector space $\left(G_{1}, W\right)$ of (single) division algebra appears in the induction process, we have to know the principal part of the zeta function for this case. This function is essentially the same as that of Godement-Jacquet [7]. In this subsection we review the principal part of the zeta function in this case.

We put $P_{1}(x)=\mathcal{N}(x)$ for $x \in W$ and $W^{\text {ss }}=\left\{x \in W \mid P_{1}(x) \neq 0\right\}$. Note that $W_{k}^{\text {ss }}=\left\{x \in W_{k} \mid x \neq 0\right\}$. The group $\mathbb{R}_{+} \times G_{1 \mathbb{A}}^{0}$ acts on $W_{\mathbb{A}}$ by assuming that $\lambda \in \mathbb{R}_{+}$ acts by multiplication by $\underline{\lambda}$. For any subset $L \subset W_{k}$, we define $\Theta_{L}\left(\Psi, \underline{\lambda} g_{1}\right)$ in the obvious manner.

Definition 4.19. For $\Psi \in \mathcal{S}\left(W_{\mathbb{A}}\right)$ and $s \in \mathbb{C}$,

$$
\begin{aligned}
Z_{W}(\Psi, s) & =\int_{\mathbb{R}_{+} \times G_{1 \mathbb{A}}^{0} / G_{1 k}} \lambda^{s} \Theta_{W_{k}^{\mathrm{ss}}}\left(\Psi, \underline{\lambda} g_{1}\right) d^{\times} \lambda d g_{1}^{0}, \\
Z_{W+}(\Psi, s) & =\int_{\substack{\mathbb{R}_{+} \times G_{1 \mathbb{A}}^{0} / G_{1 k} \\
\lambda \geq 1}} \lambda^{s} \Theta_{W_{k}^{\mathrm{ss}}}\left(\Psi, \underline{\lambda} g_{1}\right) d^{\times} \lambda d g_{1}^{0} .
\end{aligned}
$$


The following lemma is a direct consequence of Lemma 4.1.

Lemma 4.20. The integral defining $Z_{W}(\Phi, s)$ converges absolutely and locally uniformly in the region $\Re(s)>m$, and the integral defining $Z_{W+}(\Phi, s)$ is an entire function.

For $x, y \in W$, we put

$$
[x, y]_{W}=\mathcal{T}(x y)
$$

This defines a non-degenerate bilinear form on $W$. We note that this bilinear form satisfies $\left[g_{1} x, g_{1}^{\iota} y\right]_{W}=[x, y]_{W}$ where we put $\left(g_{11}, g_{12}\right)^{\iota}=\left(g_{12}^{-1}, g_{11}^{-1}\right)$.

We define the Fourier transform on $\mathcal{S}\left(W_{\mathbb{A}}\right)$ by

$$
\Psi^{*}(x)=\int_{W_{\mathbb{A}}} \Psi(y)\left\langle[x, y]_{W}\right\rangle d y .
$$

Then by the Poisson summation formula, we have

$$
\Theta_{W_{k}^{\mathrm{ss}}}\left(\Psi, \underline{\lambda} g_{1}\right)=\lambda^{-m} \Theta_{W_{k}^{\mathrm{ss}}}\left(\Psi^{*}, \underline{\lambda}^{-1}\left(g_{1}\right)^{\iota}\right)+\lambda^{-m} \Psi^{*}(0)-\Psi(0) .
$$

Applying the above equation, we obtain the following principal part formula for this zeta function.

Proposition 4.21. We have

$$
Z_{W}(\Psi, s)=Z_{W+}(\Psi, s)+Z_{W+}\left(\Psi^{*}, m-s\right)+\tau\left(G_{1}\right)\left(\frac{\Psi^{*}(0)}{s-m}-\frac{\Psi(0)}{s}\right),
$$

where $Z_{W+}(\Psi, s)$ and $Z_{W+}\left(\Psi^{*}, m-s\right)$ are entire functions.

$\S$ 4.7. Contribution from unstable strata. In this subsection, we express the residue of $\Xi_{1}(\Phi, w)$ in terms of $Z_{W}$ defined in the previous subsection. We identify $Y_{1}$ (see \$4.4) with the space $W$ of single division algebras in 4.6 .

Definition 4.22. For $\Phi \in \mathcal{S}\left(V_{\mathbb{A}}\right)$, we define a Schwartz-Bruhat function $\mathcal{R}_{W} \Phi$ on $W_{\mathbb{A}}$ by restricting to $Y_{1 \mathbb{A}}$.

Proposition 4.23. By changing $\psi$ if necessary, we have

$$
\Xi_{1}(\Phi, w) \sim \rho \Lambda(w ; 1) Z_{W}\left(\mathcal{R}_{W} \Phi, 2\right) .
$$

Proof. We have

$$
\begin{aligned}
\Xi_{1}(\Phi, w) & =\int_{G_{\mathbb{A}}^{0} / G_{k}} \Theta_{S_{1 k}}\left(\Phi, g^{0}\right) \mathcal{E}\left(g^{0}, w\right) d g^{0} \\
& =\int_{G_{\mathbb{A}}^{0} / P_{k}} \Theta_{Y_{1 k}^{\mathrm{ss}}}\left(\Phi, g^{0}\right) \mathcal{E}\left(g^{0}, w\right) d g^{0} \\
& =\int_{P_{\mathbb{A}}^{0} / P_{k}} \Theta_{Y_{1 k}^{\mathrm{ss}}}\left(\Phi, p^{0}\right) \mathcal{E}\left(p^{0}, w\right) d g^{0} \\
& =\int_{G_{1 \mathbb{A}}^{0} / G_{1 k} \times B_{2 \mathbb{A}}^{0} / B_{2 k}} \Theta_{Y_{1 k}^{\mathrm{ss}}}\left(\Phi,\left(g_{1}, b_{2}\right)\right) \mathcal{E}\left(b_{2}, w\right) d g_{1} d b_{2} \\
& =\int_{G_{1 \mathbb{A}}^{0} / G_{1 k} \times T_{2 \mathbb{A}}^{0} / T_{2 k}} \mu^{2} \Theta_{Y_{1 k}^{\mathrm{ss}}}\left(\Phi,\left(g_{1}, t_{2}\right)\right) \mathcal{E}_{N_{2}}\left(t_{2}, w\right) d g_{1} d^{\times} t_{2} .
\end{aligned}
$$

The last step is because $N_{2}$ acts on $Y_{1}$ trivially. By changing $g_{11}$ to $g_{11} t_{22}^{-1}$, we have

$$
\Xi_{1}(\Phi, w)=\int_{\mathbb{R}_{+} \times G_{1 \mathbb{A}}^{0} / G_{1 k}} \mu^{2} \Theta_{W_{k}^{\mathrm{ss}}}\left(\mathcal{R}_{W} \Phi,\left(\mu, g_{1}\right)\right) \mathcal{E}_{N_{2}}\left(a\left(\underline{\mu}^{-1}, \underline{\mu}\right), w\right) d^{\times} \mu d g_{1} \text {. }
$$


By the definition of $Z_{W}(\Psi, s)$, we have

$$
\int_{\mathbb{R}_{+} \times G_{1 \mathbb{A}}^{0} / G_{1 k}} \mu^{\mp s+1} \Theta_{W_{k}^{\text {ss }}}\left(\mathcal{R}_{W} \Phi,\left(\mu, g_{1}\right)\right) d^{\times} \mu d g_{1}=Z_{W}\left(\mathcal{R}_{W} \Phi, \mp s+1\right)
$$

for $\Re(s)<-3, \Re(s)>3$, respectively. Since

$$
\frac{1}{2 \pi \sqrt{-1}} \int_{\Re(s)=r_{2}<-3} Z_{W}\left(\mathcal{R}_{W} \Phi,-s+1\right) \Lambda(w ; s) d s \sim 0,
$$

we have

$$
\Xi_{1}(\Phi, w) \sim \frac{1}{2 \pi \sqrt{-1}} \int_{\Re(s)=r_{3}>3} Z_{W}\left(\mathcal{R}_{W} \Phi, s+1\right) \phi(s) \Lambda(w ; s) d s .
$$

By Proposition 4.21, $Z_{W}\left(\mathcal{R}_{W} \Phi, s+1\right)$ has a simple pole at $s=3$ and is holomorphic for $\Re(s)>0$ except for that point. If we consider $(s-3) \psi(s)$ instead of $\psi(s)$, this function still satisfies the property we have assumed. Namely,

$$
\sup _{c_{1}<\Re(s)<c_{2}}\left(1+|s|^{N}\right)|(s-3) \psi(s)|<\infty
$$

for all $c_{1}<c_{2}, N>0$ and $\left.(s-3) \psi(s)\right|_{s=1} \neq 0$. Therefore, by changing $\psi(s)$ to $(s-3) \psi(s)$, we may assume that $Z_{W}\left(\mathcal{R}_{W} \Phi, s+1\right) \Lambda(w ; s)$ is holomorphic for $\Re(s)>0$. (This is the passing principle (3.6.1) of [20].) Hence,

$$
\begin{aligned}
\Xi_{1}(\Phi, w) \sim & \frac{1}{2 \pi \sqrt{-1}} \int_{\Re(s)=1 / 2} Z_{W}\left(\mathcal{R}_{W} \Phi, s+1\right) \phi(s) \Lambda(w ; s) d s \\
& +\varrho \Lambda(w ; 1) Z_{W}\left(\mathcal{R}_{W} \Phi, 2\right) \\
& \sim \varrho \Lambda(w ; 1) Z_{W}\left(\mathcal{R}_{W} \Phi, 2\right)
\end{aligned}
$$

This proves the proposition.

\section{$\S$ 4.8. The principal part formula.}

Theorem 4.24. Suppose that $\Phi=\mathrm{M} \Phi$. Then

$$
\begin{aligned}
Z(\Phi, s)= & Z_{+}(\Phi, s)+Z_{+}(\widehat{\Phi}, 2 m-s) \\
& +\tau\left(G_{1}\right) \mathfrak{V}_{2}\left(\frac{\widehat{\Phi}(0)}{s-2 m}-\frac{\Phi(0)}{s}\right)+\frac{Z_{W}\left(\mathcal{R}_{W} \widehat{\Phi}, 2\right)}{s-(2 m-2)}-\frac{Z_{W}\left(\mathcal{R}_{W} \Phi, 2\right)}{s-2},
\end{aligned}
$$

where the first two terms in the right hand side are entire functions.

Proof. By Proposition 4.17, 4.18 and 4.23.

$$
I^{0}(\Phi, w)=\rho \Lambda(w ; 1)\left(\tau\left(G_{1}\right) \mathfrak{V}_{2}(\widehat{\Phi}(0)-\Phi(0))+Z_{W}\left(\mathcal{R}_{W} \Phi, 2\right)-Z_{W}\left(\mathcal{R}_{W} \widehat{\Phi}, 2\right)\right)
$$

for a suitable choice of $\psi(s)$. Hence, together with Proposition 4.15, we obtain

$$
I^{0}(\Phi)=\tau\left(G_{1}\right) \mathfrak{V}_{2}(\widehat{\Phi}(0)-\Phi(0))+Z_{W}\left(\mathcal{R}_{W} \Phi, 2\right)-Z_{W}\left(\mathcal{R}_{W} \widehat{\Phi}, 2\right) .
$$

Recall that $I(\Phi, s)=\int_{0}^{1} I^{0}\left(\Phi_{\lambda}\right) d^{\times} \lambda$ where $\Phi_{\lambda}(x)=\Phi(\underline{\lambda} x)$. It is easy to see that

$$
\Phi_{\lambda}(0)=\Phi(0), \quad \widehat{\Phi_{\lambda}}(0)=\lambda^{-2 m} \widehat{\Phi}(0) .
$$

Since

$$
\begin{aligned}
& Z_{W}\left(\mathcal{R}_{W} \Phi_{\lambda}, s\right)=\lambda^{-s} Z_{W}\left(\mathcal{R}_{W} \Phi, s\right) \\
& Z_{W}\left(\mathcal{R}_{W} \widehat{\Phi_{\lambda}}, s\right)=\lambda^{2 m-s} Z_{W}\left(\mathcal{R}_{W} \widehat{\Phi}, s\right)
\end{aligned}
$$


we get

$$
\begin{aligned}
& Z_{W}\left(\mathcal{R}_{W} \Phi_{\lambda}, 2\right)=\lambda^{-2} Z_{W}\left(\mathcal{R}_{W} \Phi, 2\right), \\
& Z_{W}\left(\mathcal{R}_{W} \widehat{\Phi_{\lambda}}, 2\right)=\lambda^{2 m-2} Z_{W}\left(\mathcal{R}_{W} \widehat{\Phi}, 2\right) .
\end{aligned}
$$

Then the theorem follows by integrating $\lambda^{s} I^{0}\left(\Phi_{\lambda}\right)$ over $s \in(0,1]$.

Theorem 1.2 in the introduction immediately follows from the above theorem.

\section{REFERENCES}

[1] N. Bourbaki. Algèbre. Éléments de mathématique. Hermann, Paris, 1958.

[2] B. Datskovsky. A mean value theorem for class numbers of quadratic extensions. Contemporary Mathematics, 143:179-242, 1993.

[3] B. Datskovsky and D.J. Wright. The adelic zeta function associated with the space of binary cubic forms II: Local theory. J. Reine Angew. Math., 367:27-75, 1986.

[4] B. Datskovsky and D.J. Wright. Density of discriminants of cubic extensions. J. Reine Angew. Math., 386:116-138, 1988.

[5] H. Davenport and H. Heilbronn. On the density of discriminants of cubic fields I. Bull. London Math. Soc., 1:345-348, 1961.

[6] H. Davenport and H. Heilbronn. On the density of discriminants of cubic fields. II. Proc. Royal Soc., A322,:405-420, 1971.

[7] R. Godement and H. Jacquet. Zeta Functions of Simple Algebras, volume 260 of Lecture Notes in Mathematics. Springer-Verlag, Berlin, Heidelberg, New York, 1972.

[8] A.C. Kable and A. Yukie. The mean value of the product of class numbers of paired quadratic fields, I. Tohoku Math. J., 54:513-565, 2002.

[9] A.C. Kable and A. Yukie. The mean value of the product of class numbers of paired quadratic fields, II. J. Math. Soc. Japan, 55:739-764, 2003.

[10] A.C. Kable and A. Yukie. The mean value of the product of class numbers of paired quadratic fields, III. J. Number Theory, 99:185-218, 2003.

[11] D. Mumford. Lectures on curves on an algebraic surface, volume 59 of Annales of Mathematical Studies. Princeton University Press, Princeton, New Jersey, 1966.

[12] H. Saito. On a classification of prehomogeneous vector spaces over local and global fields. Journal of Algebra, 187:510-536, 1997.

[13] H. Saito. Convergence of the zeta functions of prehomogeneous vector spaces. Nagoya. Math. J., 170:1-31, 2003.

[14] M. Sato and T. Kimura. A classification of irreducible prehomogeneous vector spaces and their relative invariants. Nagoya Math. J., 65:1-155, 1977.

[15] M. Sato and T. Shintani. On zeta functions associated with prehomogeneous vector spaces. Ann. of Math., 100:131-170, 1974.

[16] T. Shintani. On Dirichlet series whose coefficients are class-numbers of integral binary cubic forms. J. Math. Soc. Japan, 24:132-188, 1972.

[17] A. Weil. Basic number theory. Springer-Verlag, Berlin, Heidelberg, New York, 1974.

[18] D.J. Wright. The adelic zeta function associated to the space of binary cubic forms part I: Global theory. Math. Ann., 270:503-534, 1985.

[19] D.J. Wright and A. Yukie. Prehomogeneous vector spaces and field extensions. Invent. Math., 110:283-314, 1992.

[20] A. Yukie. Shintani Zeta Functions, volume 183 of London Math. Soc. Lecture Note Series. Cambridge University Press, Cambridge, 1993.

[21] A. Yukie. On the Shintani zeta function for the space of pairs of binary Hermitian forms. J. Number Theory, 92:205-256, 2002.

Graduate School of Mathematical Sciences, University of Tokyo, 3-8-1 Komaba MEGORO-KU, TOKYO 153-0041, JAPAN

E-mail address: tani@ms.u-tokyo.ac.jp 